Proceedings of the 2012 Winter Simulation Conference

C. Laroque, J. Himmelspach, R. Pasupathy, O. Rose, and A.M. Uhrmacher, eds

\title{
SIMULATION-BASED DISTRIBUTED FUZZY CONTROL FOR WIP IN A MULTI-VARIETY AND SMALL-BATCH DISCRETE PRODUCTION SYSTEM WITH ONE TIGHTLY COUPLED CELL
}

\author{
Run Zhao \\ Soemon Takakuwa \\ Graduate School of Economics and Business Administration \\ Nagoya University \\ Furo-cho, Chikusa-ku, Nagoya, Aichi 464-8601, JAPAN
}

\begin{abstract}
This paper examines a multi-variety and small-batch production system with a tightly coupled cell. Using production data analysis, various random factors and constraints in a system with a tightly coupled bottleneck cell caused higher work-in-process (WIP) inventory levels and longer cycle times. Aiming to resolve these production problems, a two-dimensional distributed fuzzy controller with two correction factors has been developed. This heuristic approach is used to supervise the dynamic WIP inventory level changes and regulate the processing rate of each workstation with simple representations and linguistic IF-THEN rules. Based on consideration of certain major stochastic factors, a simulation model is explored with a control objective to maintain the WIP and cycle time at a low level. Simulation results show that this optimized control policy avoids system imbalances and eliminates bottlenecks. By comparison, the proposed approach significantly improves the system's performance and robustness.
\end{abstract}

\section{INTRODUCTION}

For modern environment-oriented manufacturing, many mechanical manufacturing enterprises have applied multi-variety and small-batch production systems with coupled cells, represented by robot agent sets to improve flexibility and precision. This production mode satisfies the diversified demands of consumers and rapid responses to market needs. In this advanced production system, work-in-process (WIP) buffers are set among workstations. They are used to balance production rhythm and guarantee production lines stability by avoiding "block"/“starvation" caused by many random events (Tao et al. 2008; Tsourveloudis 2010; Luca 2011). However, a high WIP inventory level leads to the following serious problems: (1) having too much liquid capital without any profit (Kenneth 1992); (2) increasing production cycle time and decreasing market responsiveness (Tsourveloudis et al. 2000); (3) requiring more space for layout; and (4) causing production imbalance. A control policy for reducing WIP, which is associated with shorter cycle times and higher productivity, is thus an important and urgent issue in modern production research.

During the production process, changing and adjusting for various stochastic factors related to WIP complicate production management. Many scholars have recently researched these WIP control policy problems in unreliable production systems. Yang et al. presented a simulation optimization approach to resolve a constant work-in-process strategy problem (Yang et al. 2007; Jan and Anders 2009). Diamantidis and Papadopoulos (2006) adopted a Markov chain model to effectively analyze WIP buffer capacity. Bai and Gershwin (1994a and 1994b) introduced a WIP control algorithm for scheduling single and multiple part-type production lines. Morteza et al. (2011) developed a new economic production quantity to consider WIP inventory and sell non-repairable imperfect products at a reduced price. Tamani et al. presented artificial intelligence-based methods for WIP control of realistic continuous manufacturing sys- 


\section{Zhao and Takakuwa}

tems (Ioannidis et al. 2004; Tamani et al. 2009; Tsourveloudis 2010). These studies mainly focused on WIP minimization or optimization by improving production scheduling or adjusting production capacity based on certain system structure hypotheses. However, research on multi-variety and small-batch discrete production systems considering major production uncertainty factors and control policies for WIP by analyzing dynamic inventory level changes is rare, especially research identifying bottlenecks caused by tightly coupled cells to design a WIP control policy.

Most current studies on production research have also performed minimal work on heuristic control policy because accurate analytical solutions are not easily attainable (Gershwin 2000). This paper thus developed a distributed heuristic fuzzy control method for a multi-variety and small-batch production system with one tightly coupled cell. A corresponding simulation model applying this optimized control approach was constructed, which considers specific major random factors and system bottlenecks. The control objective involves keeping WIP inventory and cycle time at low levels while improving productivity by dynamically regulating the processing rate according to inventory-level changes of distributed WIP buffers between workstations. Finally, simulation results were presented along with comparisons and remarked to validate the effectiveness of the proposed approach.

\section{APPROACH}

\subsection{Approach Review}

In a production system viewed as a network of workstations and buffers, analytical results have demonstrated the superiority of surplus-based systems (Ioannidis et al. 2004). In surplus-based systems, control policy is determined based on how far cumulative or in-process production is ahead of or behind a certain hedging-point. The case study in this paper is also considered as a surplus-based system. The production rate for each workstation is adjusted by investigating whether the real-time WIP inventory level is higher or lower than a hedging point. An actual production system is a black-box system that is synthetically restricted by various random factors. Accurately controlling these variable factors to achieve predetermined objectives is more difficult, and NP-hard problems are frequently encountered. A heuristic control policy has thus been gradually considered to achieve a satisfactory strategy that is not an exact solution (Gershwin 2000). Consequently, this paper develops a heuristic fuzzy control method. The demonstrated advantages of this control method are that it is computationally simple and can be applied in real-time and dynamic control/scheduling (Tsourveloudis et al. 2007). Applying this approach can avoid disturbances from bottlenecks caused by a tightly coupled cell and achieve lower WIP inventory levels, shorter cycle time and higher productivity.

The fuzzy control method has been applied in production systems by Tamani et al., resulting in successful heuristic fuzzy production control applications for WIP (Tsourveloudis et al. 2000; Tamani et al. 2009; Tsourveloudis 2010; Tamani et al. 2011). Additionally, their research achievements and successful applications provide many suggestions and references. For easier analysis, their study cases are simulated by viewing the production system as a continuous system while only considering two random factors: machine failure/repair probability and demand change. However, the most realistic mechanical manufacturing system is a classic discrete system. This is especially apparent in a multi-variety and small-batch production system because various stochastic factors cause random WIP changes and reduce system performance. A bottleneck in a system with high "block"/"starvation" frequency can also disturb the whole discrete system. Based on the studies of Tamani et al., we consider this study case as a discrete system with more uncertain factors, and provide a closer representation of the actual production system. This paper also further improves the distributed fuzzy control method by increasing two correction factors, which can easily and quickly control the system's performance, compared to the methods used by Tamani et al. (Tamani et al. 2009; Tamani et al. 2011). 


\section{Zhao and Takakuwa}

\subsection{Fuzzy Control Method}

An appropriate control policy for a production system can satisfy multiple conflicting criteria and adapt to dynamic and stochastic constraints. A fuzzy logic controller uses a mathematical structure and method to control the production operation with some simple control principle representations using IF-THEN rules. This paper applied a two-dimensional (double-input and simple-output) fuzzy logic controller with two correction factors. For on-site supervisors, the easiest way to adopt a control strategy that regulates processing rates is to investigate the relative and absolute error values in WIP inventory levels for each distributed workstation in each check time interval. According to a surplus-based system, the relative error value is the difference between the actual WIP value and a hedging point. The absolute error value refers to the difference between successive WIP values. These relative and absolute error values constitute the double input for the fuzzy logic controller, and the processing rate is the simple output. For the two correction factors, the first factor is set to quickly eliminate errors when the actual WIP inventory levels drastically depart from the hedging point, and the second factor maintains stability when the actual WIP inventory level is near the hedging point. The other main inputs affecting the system output are dynamic and stochastic factors, which can cause discrete WIP level and system performance changes. Figure 1 shows a two-dimensional fuzzy logic structure with two correction factors.

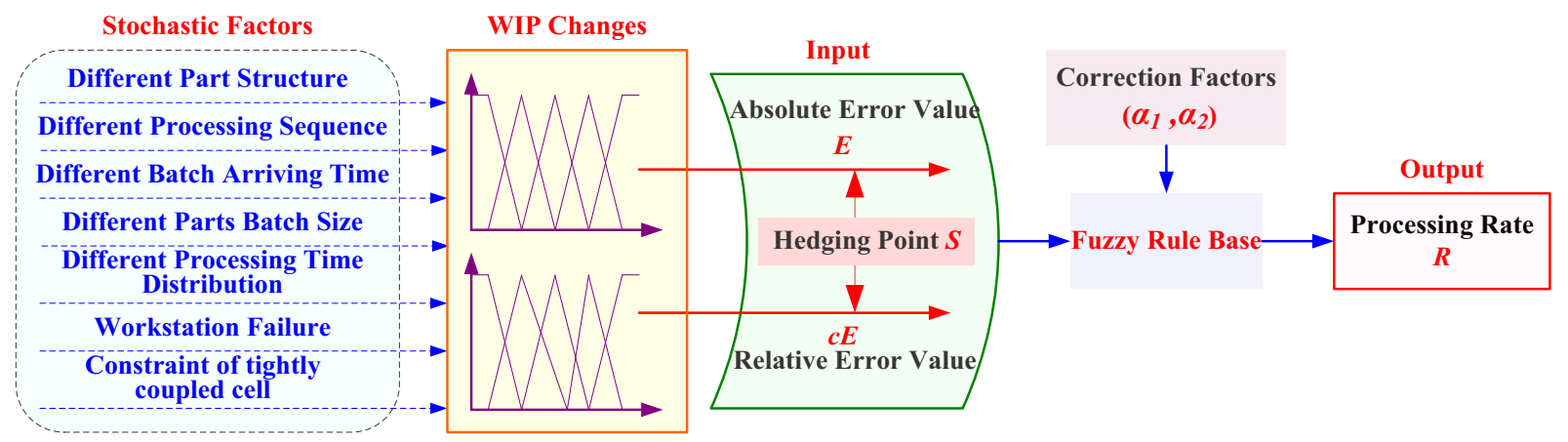

Figure 1: A double-input and simple-output fuzzy logic structure with two correction factors.

For fuzzy logic controllers, a control policy is described using linguistic IF-THEN rules with appropriate mathematical meanings (Driankov 1994). For the double-input and simple-output fuzzy logic controller in this work, the rule base for the control model contains rules with the following form:

\section{Theorem 1 IF $\boldsymbol{E}$ is $\boldsymbol{X} A N D$ cE is $\boldsymbol{Y}$, THEN $\boldsymbol{R}$ is $\boldsymbol{Z}$}

Here, $\boldsymbol{E}$ and $\boldsymbol{c} \boldsymbol{E}$ are the inputs' relative and absolute WIP error values, respectively. $\boldsymbol{R}$ is the output or processing rate. These inputs and output are divided into five corresponding linguistic variations sets: $\boldsymbol{X}=\boldsymbol{Y}=\boldsymbol{Z}=\{\boldsymbol{P L}$ (Positive Large), $\boldsymbol{P S}$ (Positive Small), $\boldsymbol{O}$ (Zero), $\boldsymbol{N L}$ (Negative Large), $\boldsymbol{N} \boldsymbol{S}$ (Negative Small) $\}$.

The correction factors, $\boldsymbol{\alpha}_{1}$ and $\boldsymbol{\alpha}_{2}$, are real numbers between 0 and 1, with $\boldsymbol{\alpha}_{1}<\boldsymbol{\alpha}_{2}$. The analytical expression for the fuzzy controller is corrected thus:

Theorem 2 IF $\boldsymbol{E} \in\{\boldsymbol{P L}, \boldsymbol{P S}\}, T H E N \boldsymbol{R}=-\left[\boldsymbol{\alpha}_{1} \times \boldsymbol{E}+\left(1-\boldsymbol{\alpha}_{1}\right) \times \boldsymbol{E C}\right]$

$$
\text { ELSEIF } \boldsymbol{E} \in\{\boldsymbol{N L}, \boldsymbol{N} \boldsymbol{S}\}, T H E N \boldsymbol{R}=-\left[\boldsymbol{\alpha}_{2} \times \boldsymbol{E}+\left(1-\boldsymbol{\alpha}_{2}\right) \times \mathbf{E C}\right]
$$

The outputs of the activated rules are aggregated to form the value of the overall control output with two correction factors, which are then defuzzified into a crisp number $\boldsymbol{Z}$.

In this paper, the processing time for each workstation is regulated based on a processing speed change rate $\boldsymbol{r}_{i}$, which is the fuzzy controller output and can be calculated by defuzzification. A VBA module in the simulation model operates this calculation, as illustrated in Section 4. 


\section{Zhao and Takakuwa}

\section{CASE STUDY}

This paper considers a case of a certain multi-variety and small-batch discrete production system with one tightly coupled cell. This system is located in an engine component manufacturing workshop of a Japanese company. Considering the system characteristics and ensuring the current logical structure, Figure 2 shows a simplified layout model for this production system. This production system mainly comprises 21 workstations and three main part families (Part Type A, B, and C), which are processed by different production lines that can use the same machines, according to their technology groups. Each part batch that enters the system includes three types of parts in random proportions. The WIP buffers are used to balance the machining capabilities, improve production stationarity, and meet the processing demands for the diversified part types. The processing capacity of each independent process in a workstation is handled by a machining center that can be controlled by regulating processing time. According to the part family characteristics, each workstation completes the processing task for various parts in a corresponding part family, either in whole or part. The processing time for each part on each workstation is different. Furthermore, there are three coupled cells: two cells are loose coupled cells with machines that act like conveyors and can do continuous processing; the remaining cell is a tightly coupled cell in the NC station, in which a robot agent is used to accurately transfer parts between two fine machines. The buffer space is limited to 12. In this cell, the milling machine is easily blocked until limited buffer space becomes available. This prototype system is called the AS-IS model.

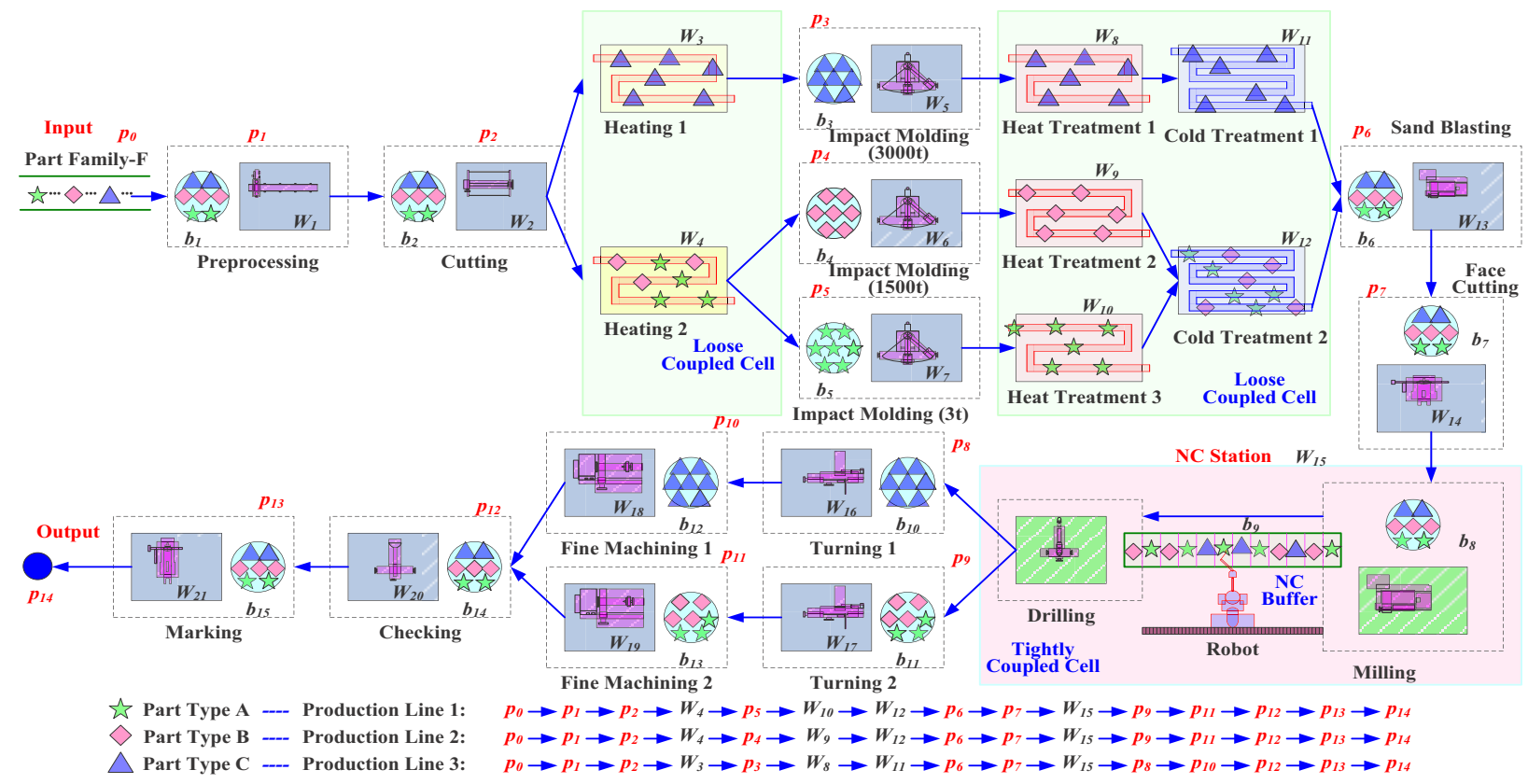

Figure 2: Multi-variety and small-batch production systems with one tightly coupled cell.

For this AS-IS model, Table 1 provides a statistical analysis of the latest two months of production data. The largest average WIP inventory level is over 80, and the standard deviations (SDs) are large. The system's ability to resist disturbance is affected by various random factors and is low. WIP control in this production system remains a serious issue. From Table 1, due to the large SD, the Takt time is unbalanced when comparing production lines. In the tightly coupled cell, the "block" frequency of the milling machine exceeds $5 \%$, but the "starvation" frequency of the drilling machine is not zero and is instead approximately $0.7 \%$. The SD of each downstream WIP level in this cell is larger than the upstream WIP. This suggests that this cell has a large effect on disturbances in the entire system and is the main bottleneck for the whole production system. 


\section{Zhao and Takakuwa}

Table 1: Latest two-month actual production statistics.

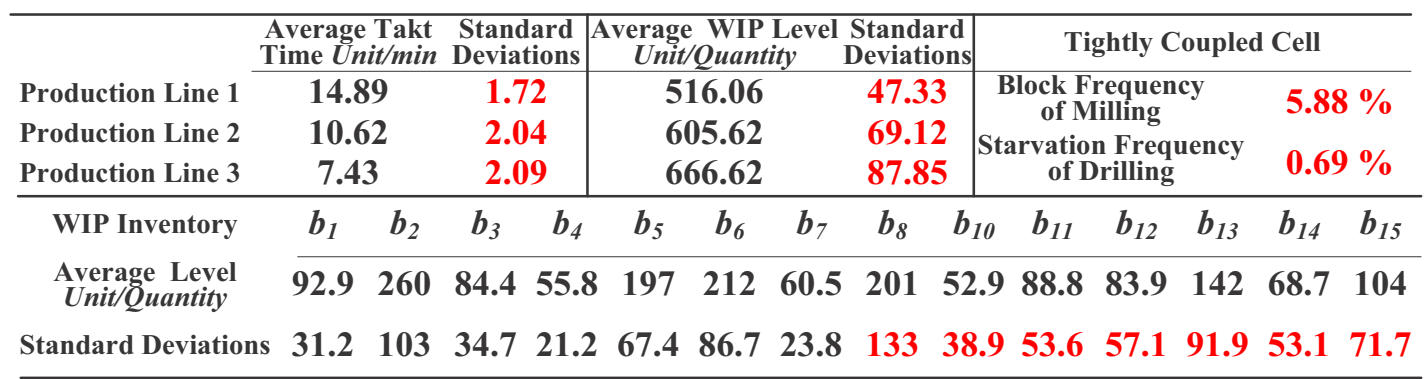

Based on data from these two months, the cycle time for each part family batch obeys a normal distribution $\left(X \sim N\left(\boldsymbol{\mu}=5220, \sigma^{2}=2700\right)\right)$. Approximately $19.31 \%$ of the batches are completed within two days, while half of the batches are completed in three days or more. Most orders thus cannot meet the delivery time of three days. Figure 3 presents the details for this distribution.

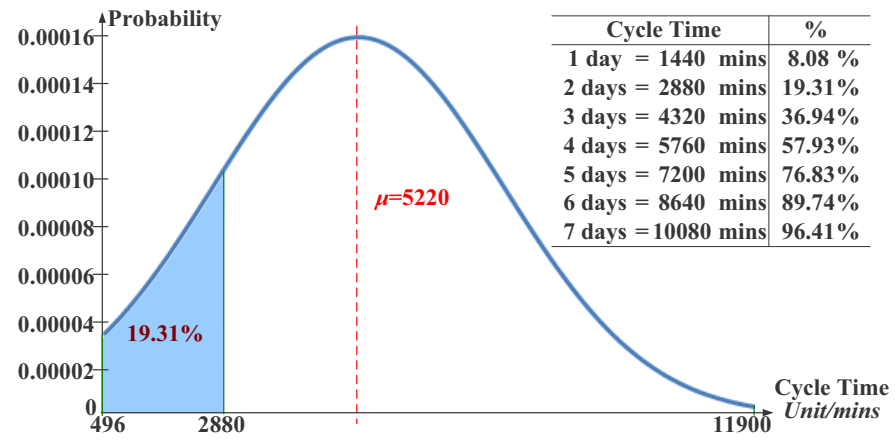

Figure 3: Probability distribution of cycle time for the AS-IS model.

Analysis and data from the AS-IS model suggest that the WIP inventory level of each workstation is high, the cycle time is long, and the tightly coupled cell seriously restricts productivity.

\section{SIMULATION}

\subsection{Simulation Submodels}

To resolve current production problems, a simulation model using an optimized fuzzy control method is developed, called the TO-BE model. To achieve the control objective and keep WIP inventory and cycle time at low levels, the simulation model comprises five sub-models, shown in Figure 4.

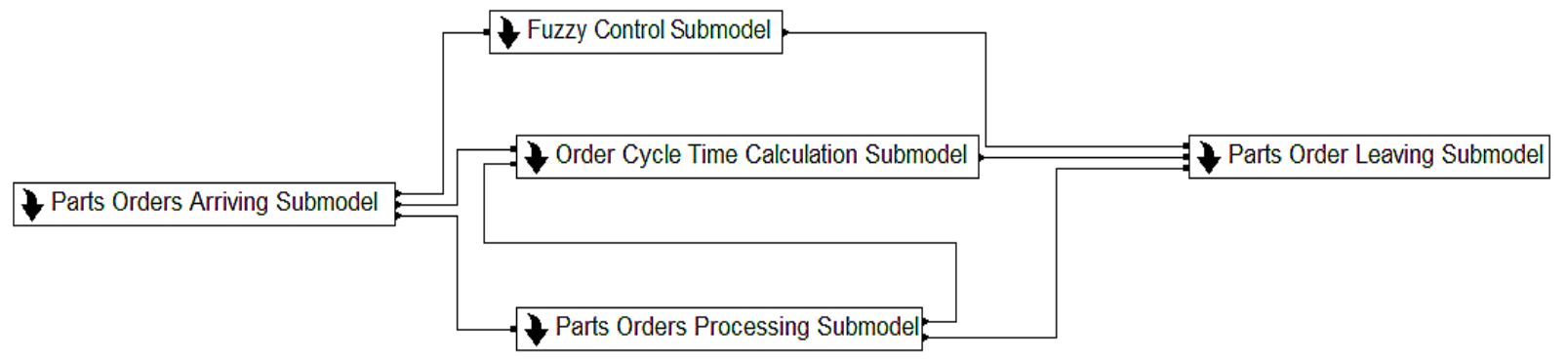

Figure 4: Simulation Submodels. 
The Parts Order Arriving sub-model is designed to simulate part family orders arriving, randomly create part batch quantities, and determine the production line. The Parts Orders Processing sub-model is designed to process parts on the corresponding workstations. The Parts Order Leaving sub-model is designed to ensure that all parts in a batch are completed and develop statistics for the parts leaving. The Fuzzy Control sub-model is the core for this simulation. It is designed to calculate changing WIP values from the Parts Orders Processing sub-model and make a control policy to regulate processing time for each workstation using a distributed fuzzy control methodology. The Order Cycle Time Calculation submodel creates WIP change statistics for workstation and processing cycle time for each part order.

\subsection{Optimized Approach-A Distributed Fuzzy Control Controller}

The Fuzzy Control sub-model embeds and uses a two-dimensional fuzzy controller with two correction factors to make an optimized control policy to regulate processing time for each distributed workstation. Because of system's stochastic changes caused by various random factors, the inventory level changes at workstations differ from one another over time. According to the distributed workstation locations in a real production system, a corresponding distributed fuzzy controller developed for each workstation simplifies making the corresponding control policy.

As Figure 5 shows, for each distributed workstation in the Parts Orders Processing sub-model, its relative and absolute WIP error values ( $\boldsymbol{e}$ and $\boldsymbol{c} \boldsymbol{e}$ ) are collected and input into the corresponding fuzzy controller in the Fuzzy Control sub-model. In this controller, the output $\boldsymbol{r}$ is computed using fuzzy processing, parameter correction, fuzzy rules selection, fuzzy inference, and defuzzification. By consulting this output, a simulation instruction based on an optimized control policy is made to regulate the processing time of a corresponding workstation.

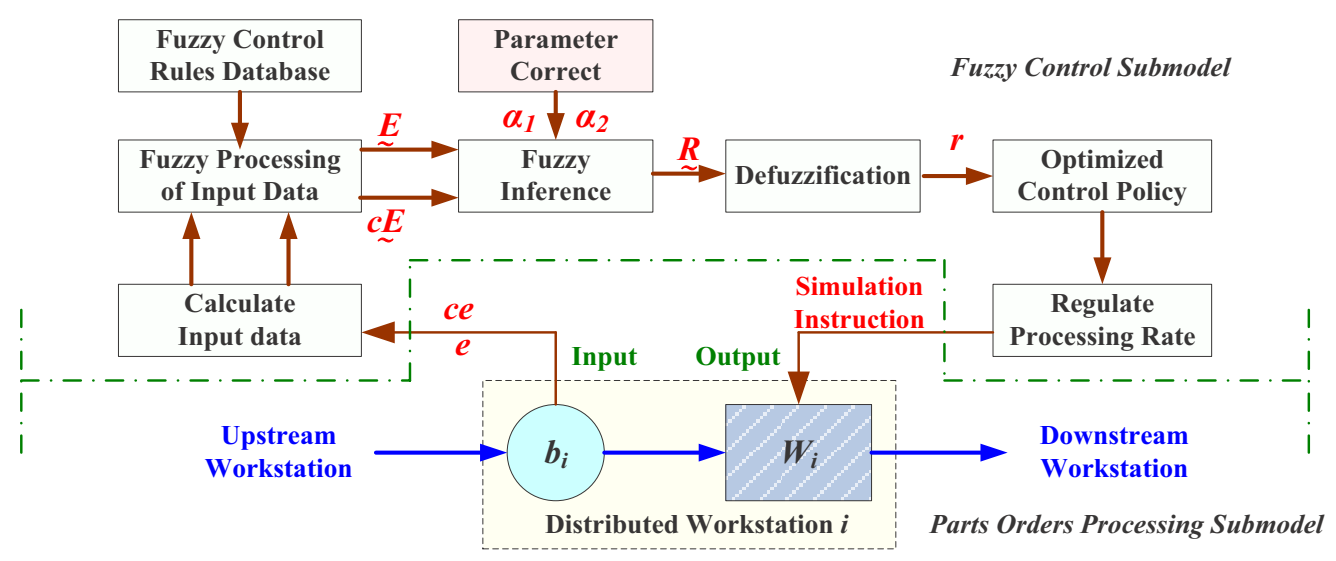

Figure 5: A distributed fuzzy controller.

In the Fuzzy Control sub-model, a VBA module operates these calculation steps during the simulation running at each check time interval. After the optimized fuzzy calculation, a control instruction is sent to the Parts Orders Processing sub-model. The process time for the corresponding workstation is regulated by $\boldsymbol{p}_{\boldsymbol{i}}$. The main steps in this optimized approach are designed as follows.

\section{[Step 1] Perform Fuzzification and Define Fuzzy Sets Universe}

The relative and absolute WIP error values ( $\boldsymbol{e}$ and $\boldsymbol{c} \boldsymbol{e})$ of each distributed workstation are input into the Fuzzy Control sub-model. The fuzzy controller converts inputs into fuzzy sets, and a quantizer $\boldsymbol{k}$ is used with $\boldsymbol{k}_{e}=\boldsymbol{k}_{c e}=1 / 5$. After fuzzification, for $\boldsymbol{e}$, three cases exist.

(1) If $\boldsymbol{e} \times \boldsymbol{k}_{\boldsymbol{e}} \in \boldsymbol{X}$, and $\mathbf{- 4}<\boldsymbol{e} \times \boldsymbol{k}_{\boldsymbol{e}}<\mathbf{4}$, it should be rounded off.

(2) If $\boldsymbol{e} \times \boldsymbol{k}_{\boldsymbol{e}} \notin X$, and $\boldsymbol{e} \times \boldsymbol{k}_{\boldsymbol{e}} \leq \mathbf{- 4}$, it should be quantized as $\mathbf{- 4}$.

(3) If $\boldsymbol{e} \times \boldsymbol{k}_{e} \notin X$, and $\boldsymbol{e} \times \boldsymbol{k}_{e} \geq \mathbf{4}$, it should be $\mathbf{4}$. 


\section{[Step 2] Design Linguistic Fuzzy Sets and Fuzzy Rules}

This paper denotes the linguistic fuzzy sets as $\boldsymbol{X}=\boldsymbol{Y}=\{\boldsymbol{P} \boldsymbol{L}, \boldsymbol{P S}, \boldsymbol{O}, \boldsymbol{N} \boldsymbol{L}, \boldsymbol{N} \boldsymbol{S}\}, \boldsymbol{\alpha}_{I}=1 / 2, \boldsymbol{\alpha}_{2}=3 / 4$. Using Theorems $1-2$, the membership function and variable assignment of inputs and the output are obtained. Membership function is a generalization of each corrected input in classical sets and represents the attribution ratio as a fuzzy set for input. After fuzzification, fuzzy rules should be made for the control policy. Figure 6 shows the results of this step.

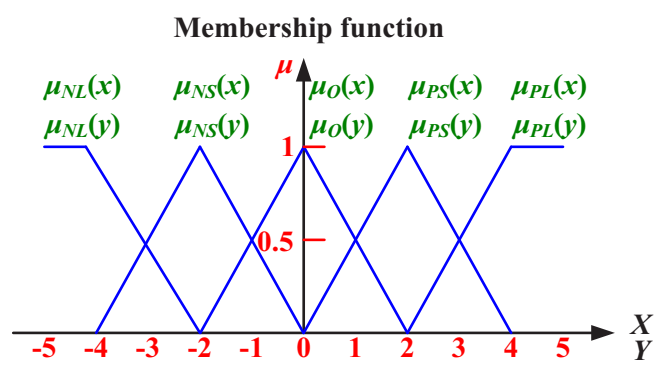

\begin{tabular}{c|ccccc}
\multicolumn{5}{c}{ Fuzzy control rules } \\
\hline \multicolumn{1}{c|}{$E C$} & $N L$ & $N S$ & $O$ & $P S$ & $P L$ \\
\hline$N L$ & $P L$ & $P L$ & $P L$ & $P S$ & $P S$ \\
$N S$ & $P L$ & $P S$ & $O$ & $O$ & $N S$ \\
$O$ & $P S$ & $O$ & $O$ & $O$ & $N S$ \\
$P S$ & $P S$ & $O$ & $O$ & $N S$ & $N L$ \\
$P L$ & $N S$ & $N S$ & $N L$ & $N L$ & $N L$ \\
\hline
\end{tabular}

Figure 6: Membership function and fuzzy control rules.

\section{[Step 3] Defuzzification}

The corrected inputs can be represented as $\boldsymbol{e}_{i}$ and $\boldsymbol{c} \boldsymbol{e}_{\boldsymbol{i}}$, with membership functions $\boldsymbol{\mu}_{X}{ }^{*}\left(\boldsymbol{e}_{\boldsymbol{i}}\right)$ and $\boldsymbol{\mu}_{\boldsymbol{X}}{ }^{*}\left(\boldsymbol{c} \boldsymbol{e}_{\boldsymbol{i}}\right)$, respectively. The membership functions of the conjunction of these two inputs, for $A N D=\min$, is:

$$
\mu_{A N D}^{*}\left(e_{i}, c e_{i}\right)=\mu_{X}^{*}\left(e_{i}\right) \wedge \mu_{Y}^{*}\left(c e_{i}\right)
$$

The following defuzzification formula gives the processing rate $\boldsymbol{r}_{\boldsymbol{i}}{ }^{*}$, which is the control degree after each WIP checking time interval:

$$
r_{i}=\frac{\sum r_{i} \mu_{Z}^{*}\left(r_{i}\right)}{\sum \mu_{Z}^{*}\left(r_{i}\right)}
$$

\section{[Step 4] Calculate the Processing Time}

Based on step 3, after setting quantizer $\boldsymbol{k}_{\boldsymbol{r}}$, the processing time $\boldsymbol{p}_{\boldsymbol{i}}$ for workstation $\boldsymbol{i}$ can be designed as the following function and regulated by $\boldsymbol{p}_{i}$ :

$$
p_{i}=p_{d} \times\left(1-r_{i} \times k_{r}\right)
$$

where, $\boldsymbol{p}_{\boldsymbol{d}}$ means the processing time created by the normal processing time distribution. After Step 3, $\boldsymbol{r}_{\boldsymbol{i}}$ has positive and negative values. $\left(\boldsymbol{r}_{\boldsymbol{i}} \times \boldsymbol{k}_{\boldsymbol{r}}\right)$ denotes the regulation value for processing time $\boldsymbol{p}_{\boldsymbol{d}}$. The process time for workstation $\boldsymbol{i}$ is regulated by $\left(1-\boldsymbol{r}_{\boldsymbol{i}} \times \boldsymbol{k}_{\boldsymbol{r}}\right)$ of the original processing time.

\subsection{Simulation Control Logic}

For the entire simulation system, dynamic testing and control of changing WIP inventory levels is the primary objective for determining a solution. However, upstream and downstream WIP buffers exist for each workstation, and the controller must select the first buffer to determine. As analyzed, the tightly coupled cell in this production system is the main bottleneck. According to the theory of constraints, this cell is viewed as the "drum" for the whole production system. The "pull" mode is thus used for the production line upstream of the "drum", and the "Push" mode is applied for the production line downstream of the "drum". Each upstream workstation of the NC station should check the downstream WIP buffer, and each downstream workstation of the NC station should check the upstream WIP buffer. This paper integrates the "Pull"/"Push" mode and the fuzzy controller into a simulation model for controlling WIP. Figure 7 shows the simulation control logic. 


\section{Zhao and Takakuwa}

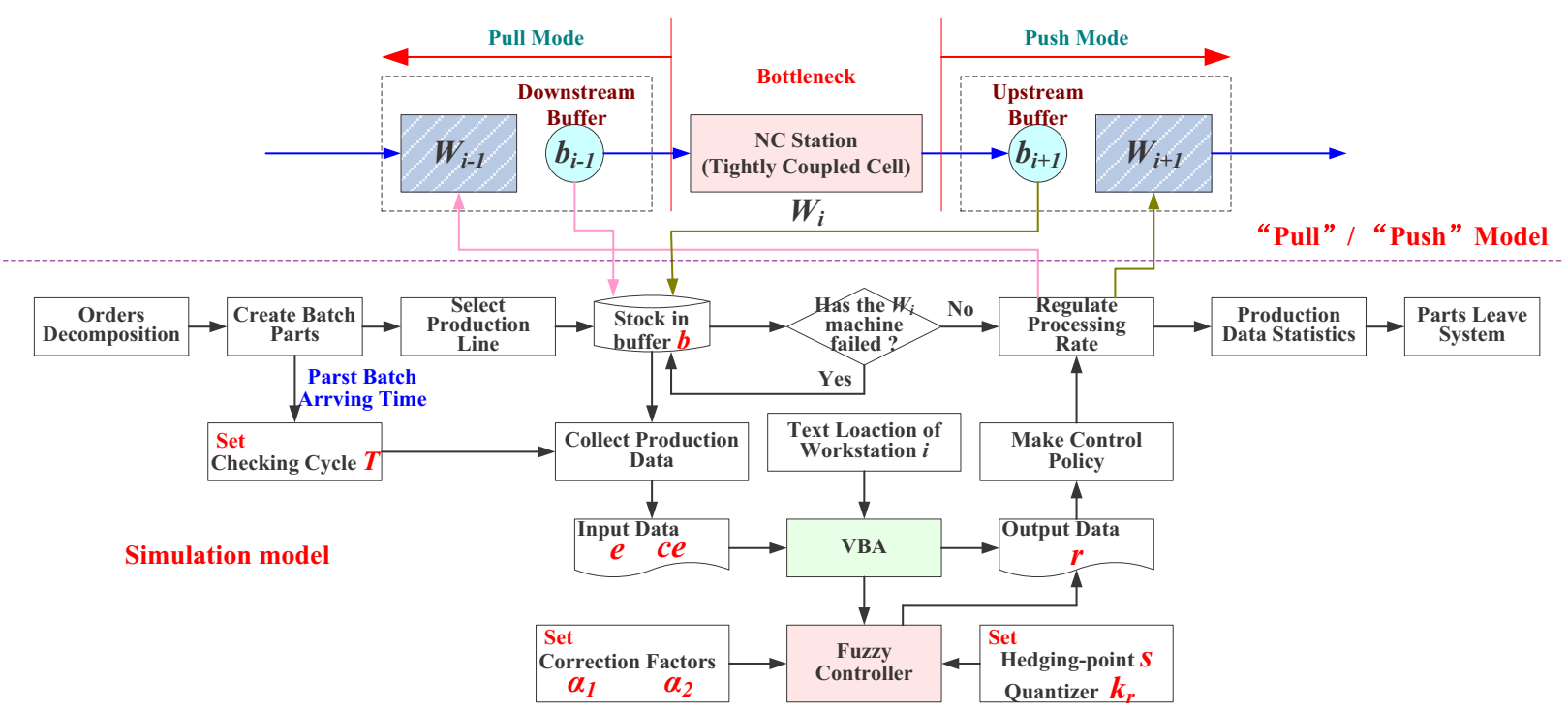

Figure 7: Simulation control logic using the optimized fuzzy control approach.

When a workstation is in the upstream line of the NC station, its downstream WIP change value is input into the corresponding distributed fuzzy controller for calculation. If the relative and absolute WIP error values are both low, the fuzzy controller makes the control policy that the productivity of this workstation should be increased. The processing time is thus reduced by simulation instruction. By doing this, the "starvation" frequency of the milling machine is reduced, and the production lines are steadier and more balanced. The NC station "pulls" the upstream line, and production performance is optimized. For a workstation downstream of the NC station, based on the same WIP value, the control policy made by the fuzzy controller is contrary to the upstream NC station line. Different outputs are made in the VBA module by distinguishing workstation location and converting the positive/negative output $\boldsymbol{r}_{\boldsymbol{i}}$ values.

\subsection{Running the Simulation}

This paper uses the Arena simulation platform to build the simulation model. To ensure simulation randomness similar to the AS-IS model with the various stochastic factors described in Figure 1, random distribution data and main parameters are set, as shown in Table 2.

Table 2: Simulation data and parameters.

\begin{tabular}{|c|c|c|c|c|c|c|c|c|c|c|}
\hline \multirow{2}{*}{$\begin{array}{l}\text { Parts Processing Time } \\
\text { Unit/min }\end{array}$} & \multirow[b]{2}{*}{ roduction Line 1} & \multirow[b]{2}{*}{ roduction Line 2} & \multirow[b]{2}{*}{ roduction Line 3} & \multicolumn{2}{|c|}{ Machine F } & Loose Coupled Cell & \multicolumn{2}{|c|}{ Run Speed } & UIII SOEC & Eengin \\
\hline & & & & Up Time & Down Time & \multirow{2}{*}{$\begin{array}{l}\text { Heating } 1\left(W_{3}\right) \\
\text { Heating } 2\left(W_{4}\right)\end{array}$} & \multicolumn{2}{|c|}{$0.72 \mathrm{~m} / \mathrm{min}$} & $0.9 \mathrm{~m}$ & $7.2 \mathrm{~m}$ \\
\hline Preprocessing $\left(W_{l}\right)$ & & & & & & & \multicolumn{2}{|c|}{$0.90 \mathrm{~m} / \mathrm{min}$} & $0.6 \mathrm{~m}$ & $7.2 \mathrm{~m}$ \\
\hline Cutting Station $\left(W_{2}\right)$ & $.9,4.1,4.3)$ & & & 20) & & \multirow{2}{*}{$\begin{array}{l}\text { Heat Treatment }\left(W_{8}\right) \\
\text { Heat Treatment }\left(W_{9}\right)\end{array}$} & \multicolumn{2}{|c|}{$0.75 \mathrm{~m} / \mathrm{min}$} & $0.9 \mathrm{~m}$ & $4.5 \mathrm{~m}$ \\
\hline 3000t Impact Molding $\left(W_{5}\right)$ & $, 11.6,14.1)$ & & & $\mathrm{XPO}(720)$ & 0) & & \multicolumn{2}{|c|}{$0.90 \mathrm{~m} / \mathrm{min}$} & $0.6 \mathrm{~m}$ & $5.4 \mathrm{~m}$ \\
\hline $1500 \mathrm{t} I$ & & TRI & & 720) & EX & Heat Treatment $\left(W_{10}\right)$ & \multicolumn{2}{|c|}{$0.90 \mathrm{~m} / \mathrm{min}$} & 0. & $5.4 \mathrm{~m}$ \\
\hline $3 t \operatorname{Imp}$ & & & & 20) & & Cold Treatment $\left(W_{11}\right)$ & \multirow{2}{*}{\multicolumn{2}{|c|}{$\begin{array}{l}0.90 \mathrm{~m} / \mathrm{min} \\
0.90 \mathrm{~m} / \mathrm{min}\end{array}$}} & & n \\
\hline Sand Blastin & TRIA & 5) & $2.5)$ & 20) & & Cold Treatment $\left(W_{12}\right)$ & & & 0. & \\
\hline Face Cut & TRIA(4.3,4.5,4.7) & TRI & TRIA & $\operatorname{EXPO}(720)$ & $\operatorname{EXPO}(30)$ & \multicolumn{5}{|c|}{ Parts Family Batch Data } \\
\hline$\left.W_{16}\right)$ & TRIA(9 & & & $\operatorname{EXPO}(720)$ & EXP & \multirow{4}{*}{\multicolumn{2}{|c|}{$\begin{array}{c}\text { Parts Type Proportion } \\
\text { Parts Batch Arriving Time } \\
\text { Unit/min } \\
\text { Each Parts Batch Quantity } \\
\text { Unit/Quantiy }\end{array}$}} & \multicolumn{3}{|c|}{$\operatorname{DISC}(0.27,1,0.61,2,1)$} \\
\hline $\begin{array}{r}\text { Turı } \\
\text { Fine Ma }\end{array}$ & TRI & & & $\begin{array}{l}\operatorname{EXPO}(720) \\
\operatorname{EXPO}(720)\end{array}$ & $\begin{array}{l}\operatorname{EXPO}(30) \\
\operatorname{EXPO}(30)\end{array}$ & & & \multicolumn{3}{|c|}{ TRIA $(360,420,480)$} \\
\hline Fine Ma & & & IM & $\operatorname{EXPO}(720)$ & $\operatorname{EXPO(30)}$ & & & \multirow{2}{*}{\multicolumn{3}{|c|}{$\operatorname{AINT}(\operatorname{TRIA}(124,138,152))$}} \\
\hline$\left(W_{21}\right)$ & TRIA & TRI & $2.8,3,3.2)$ & $\operatorname{EXPO}(720)$ & $\operatorname{EXPO}(15)$ & & & & & \\
\hline Marking $\left(W_{22}\right)$ & TRIA & TPIA & $.1,3.3,3.5)$ & $\operatorname{EXPO}(720)$ & $\operatorname{EXPO}(15)$ & \multicolumn{5}{|c|}{ Fuzzy Control Parameters } \\
\hline Tightl & & & & & & \multirow{3}{*}{\multicolumn{2}{|c|}{$\begin{array}{cc}\text { Hedging-Point } s & 30 \\
k_{e}=k_{c e} & 1 / 5 \\
k_{r} & 1 / 10\end{array}$}} & $T$ & \multicolumn{2}{|c|}{24 Hours } \\
\hline NC Sta & TRIA(4.1,4.3,4.5) & IRIA $(3.1,3.5,5.5)$ & TRIA(2.1,2.3,2.5) & \multicolumn{2}{|c|}{$\operatorname{EXPO}(1200) \operatorname{EXPO}(20)$} & & & & \multicolumn{2}{|c|}{$1 / 2$} \\
\hline NC Station-Milling $\left(W_{15}\right)$ & TRIA $(4.3,4.5,4.7)$ & TRIA(3.3,3.5,3.7) & $\operatorname{TRIA}(2.3,2.5,2.7)$ & \multicolumn{2}{|c|}{$\operatorname{EXPO(1200)} \operatorname{EXPO(20)}$} & & & $\alpha_{2}$ & & \\
\hline
\end{tabular}


The simulation duration is set to two months to match the AS-IS model. To avoid the impact of data deviation on simulation system performance due to the initial status, a steady-state simulation is appropriate for this study. According to the statistical analysis for output inventory level data based on the original simulation model, the effects of the artificial initial conditions have worn off after 5000 minutes. The warm-up period is thus selected as 5000 minutes. Twenty replications are performed to obtain good statistical analysis features and a narrower $95 \%$ prediction interval. To reduce output variance affected by random number generation, a common random number method is applied so that all simulations running in the same input have the same random situation.

\subsection{Simulation Results}

In this section, the average WIP inventory level of each distributed workstation and cycle time are dynamically calculated with the TO-BE model. Simulation results show that the largest WIP average inventory level for each workstation is under 60. Figure 8 shows that the SD is also largely reduced. Although the WIP for the preprocessing workstation is increased by 17 over the AS-IS model, the SD is reduced, which means system stability is effectively controlled.

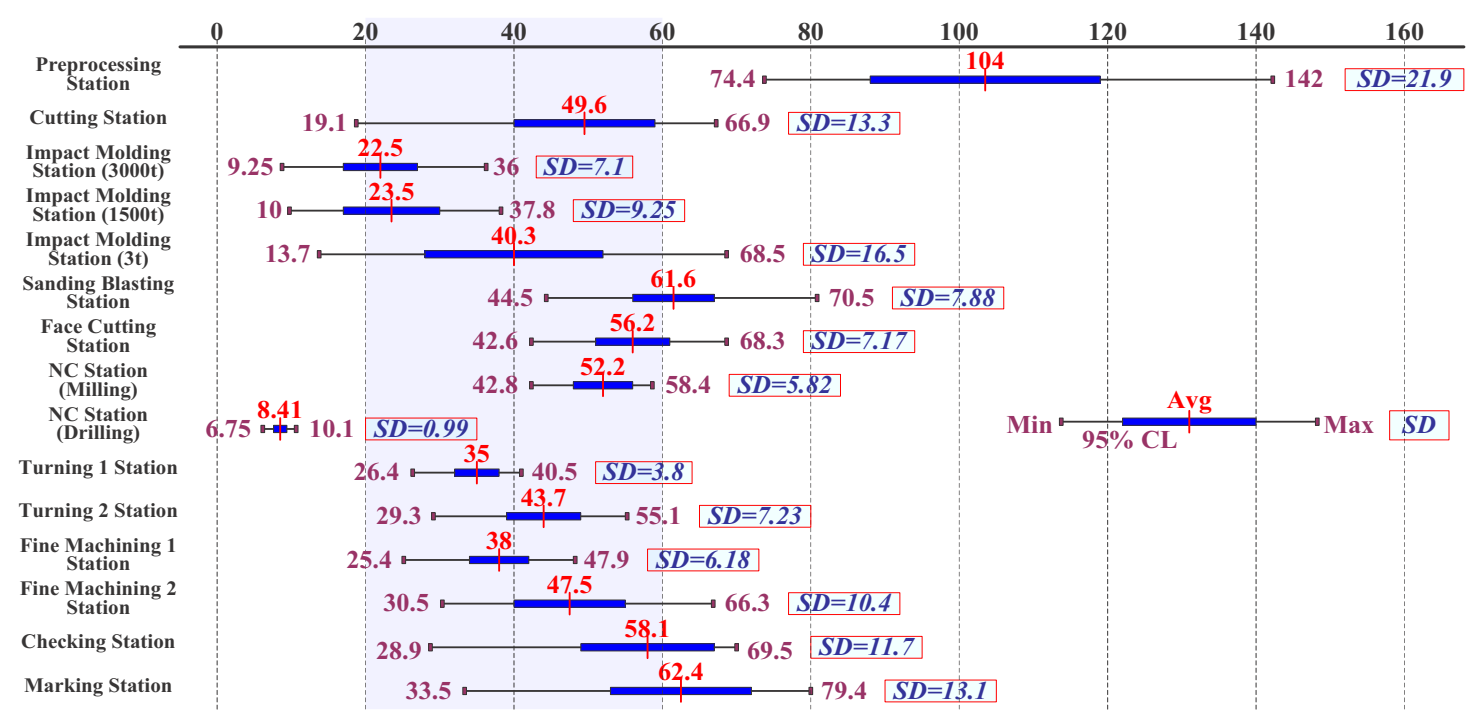

Figure 8: Statistical data of the WIP inventory for the TO-BE Model by simulation.

Figure 9 provides the cycle time for the TO-BE Model, which follows a normal distribution $\left(X_{\sim} \boldsymbol{N}\right.$ $\left.\left(\boldsymbol{\mu}=558, \sigma^{2}=87\right)\right)$. Approximately $97.81 \%$ of the batch parts can be completed before two days, and $100 \%$ of the batches are completed before three days. The delivery date is thus reduced and met.

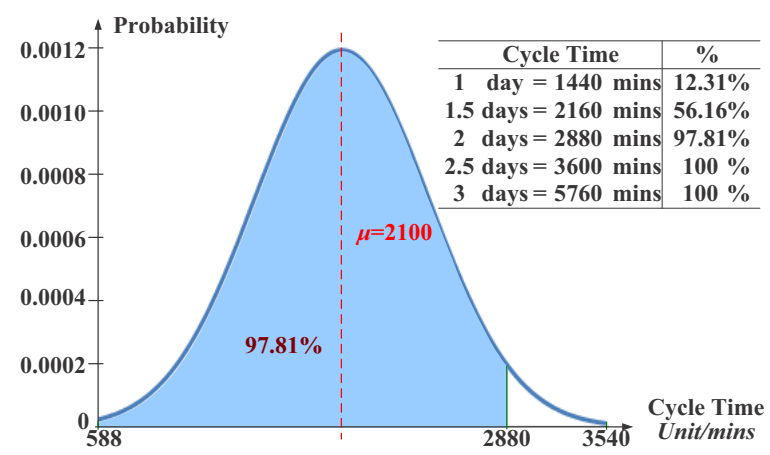

Figure 9: Probability distribution of cycle time for the TO-BE model. 


\section{Zhao and Takakuwa}

\subsection{Comparison and Remarks}

Table 3 compares the AS-IS and TO-BE models. In the TO-BE model, the average WIP inventory level of each production line has been reduced by over $60 \%$. The width of the $95 \%$ confidence interval and the SD for the TO-BE model are narrower and smaller than those for the AS-IS model, respectively. The cycle time distribution SD for the TO-BE model is considerably smaller than that for the AS-IS model. These results show that the TO-BE model has higher stability, a stronger capacity for resisting disturbance, and greater flexibility than the AS-IS model. Furthermore, the cycle time of the TO-BE model is shortened and the delivery day requirement is satisfied for almost all part batches. Conversely, for the bottleneck tightly coupled cell in the TO-BE model, simulation results show that the "block" frequency for milling is below $1 \%$, and the "starvation" frequency for drilling is approximately $0.1 \%$; effects due to the bottleneck are thus essentially eliminated.

Table 3: Comparison of the AS-IS and TO-BE models.

\begin{tabular}{|c|c|c|c|c|c|c|c|c|c|}
\hline \multirow{2}{*}{ (Unit/Quantity) } & \multicolumn{3}{|c|}{ AS-IS Model } & \multicolumn{3}{|c|}{ TO-BE Model } & \multicolumn{3}{|c|}{ Decline Ratio \% } \\
\hline & Average & Half Width & $\begin{array}{c}\text { Standard } \\
\text { Deviations }\end{array}$ & Average & Half Width & $\begin{array}{c}\text { Standard } \\
\text { Deviations }\end{array}$ & Average & Half Width & $\begin{array}{c}\text { Standard } \\
\text { Deviations }\end{array}$ \\
\hline Production Line 1 WIP & 516.06 & 16.76 & 47.33 & 202.16 & 6.3 & 18.11 & $60.83 \%$ & $62.41 \%$ & $61.74 \%$ \\
\hline Production Line 2 WIP & 605.62 & 26.59 & 69.12 & 202.81 & 7.34 & 26.70 & $66.51 \%$ & $72.40 \%$ & $61.37 \%$ \\
\hline Production Line 3 WIP & 666.62 & 29.26 & 87.85 & 232.01 & 8.41 & 28.05 & $65.20 \%$ & $71.26 \%$ & $68.07 \%$ \\
\hline $\begin{array}{c}\text { Cycle Time } \\
\text { Unit/min }\end{array}$ & \multicolumn{3}{|c|}{$X \sim N\left(\mu=5220, \sigma^{2}=2700\right)$} & \multicolumn{3}{|c|}{$X \sim N\left(\mu=2100, \sigma^{2}=387\right)$} & $59.61 \%$ & I & $85.67 \%$ \\
\hline \multirow{2}{*}{ Tightly Coupled Cell } & \multicolumn{2}{|c|}{$\begin{array}{c}\text { Block } \\
\text { Frequency }\end{array}$} & $\begin{array}{l}\text { Starvation } \\
\text { Frequency }\end{array}$ & $\begin{array}{r}\text { Blocl } \\
\text { Freques }\end{array}$ & & $\begin{array}{l}\text { tarvation } \\
\text { requency }\end{array}$ & \multirow[t]{2}{*}{$\underset{\%}{\text { Decline Ratio }}$} & $\begin{array}{c}\text { Block } \\
\text { io } \\
\text { Frequency }\end{array}$ & $\begin{array}{l}\text { Starvation } \\
\text { Frequency }\end{array}$ \\
\hline & \multicolumn{2}{|c|}{$5.88 \%$} & $0.69 \%$ & $0.71 \%$ & & $0.103 \%$ & & $87.93 \%$ & $85.07 \%$ \\
\hline
\end{tabular}

The TO-BE model using the optimized approach is developed based on the major parameters in Table 2. According to Figure 1, stochastic factors affect production system stability and the WIP changes. In this surplus-based system, the hedging point $\boldsymbol{s}$, check time interval $\boldsymbol{T}$, quantizer $\boldsymbol{k}_{\boldsymbol{r}}$, and correction factors $\boldsymbol{\alpha}_{1} / \boldsymbol{\alpha}_{2}$, can greatly disturb the production system. The setting of $\boldsymbol{s}$ thus determines the descent speed of the WIP inventory level, system balance, and selection of other fuzzy control variables.

Table 4: Robustness analysis of WIP and cycle time with variations in $\boldsymbol{s}$.

\begin{tabular}{|c|c|c|c|c|c|c|c|c|c|c|}
\hline \multirow{2}{*}{$\begin{array}{c} \\
\text { (Unit/Quantity) } \\
5\end{array}$} & \multicolumn{2}{|c|}{$\begin{array}{c}\text { Production Line } 1 \\
\text { Average WIP }\end{array}$} & \multicolumn{2}{|c|}{$\begin{array}{c}\text { Production Line } 2 \\
\text { Average WIP }\end{array}$} & \multicolumn{2}{|c|}{$\begin{array}{c}\text { Production Line } 3 \\
\text { Average WIP }\end{array}$} & \multicolumn{4}{|c|}{ Average Cycle Time } \\
\hline & 347.79 & $72.03 \%$ チ & 438.73 & $126.33 \%$ ケ & 500.56 & $115.75 \%$ ศ & 3885 & $85.00 \%$ ケ & 1623 & $319.38 \%$ ケ \\
\hline 10 & 317.68 & $57.14 \%$ & 394.47 & $94.50 \%$ & 450.23 & $94.06 \%$ & 3544 & $68.76 \%$ & 1431 & $269.77 \%$ \\
\hline 15 & 299.55 & $48.17 \%$ & 364.31 & $79.68 \%$ & 413.61 & $78.27 \%$ & 3319 & $58.05 \%$ & 1245 & $221.71 \%$ \\
\hline 20 & 211.37 & $4.56 \%$ & 238.40 & $17.54 \%$ & 271.86 & $17.18 \%$ & 2331 & $11.00 \%$ & $\mathbf{5 2 7}$ & $36.18 \%$ \\
\hline 25 & 204.68 & $1.25 \%$ & 218.59 & $7.78 \%$ & 249.70 & $7.62 \%$ & 2195 & $4.52 \%$ & 432 & $11.63 \%$ \\
\hline 30 & $202.16 \mathrm{MIN}$ & $0 \%$ & 202.81 & $0 \%$ & 232.01 & $0 \%$ & 2100 & $0 \%$ & $387 \mathrm{MIN}$ & $0 \%$ \\
\hline 35 & 206.21 & $2.00 \%$ & 195.69 & $3.51 \% \downarrow$ & 224.39 & $3.28 \%$ & 2065 MIN & $1.67 \%$ & 395 & $2.07 \%$ \\
\hline 40 & 213.09 & $5.41 \%$ & $193.41 \mathrm{MIN}$ & $4.63 \%$ & 221.74 MIN & $4.43 \%$ & 2071 & $1.38 \%$ & 412 & $6.46 \%$ \\
\hline 45 & 222.16 & $9.89 \%$ & 194.92 & $3.89 \%$ & 222.82 & $3.96 \%$ & 2104 & $1.90 \%$ & 443 & $14.47 \%$ \\
\hline 50 & 232.26 & $14.89 \%$ & 197.33 & $2.70 \% \downarrow$ & 225.56 & $2.78 \%$ & 2147 & $2.24 \%$ & 479 & $23.77 \%$ \\
\hline 55 & 242.01 & $19.71 \%$ & 229.51 & $13.17 \%$ & 295.41 & $27.33 \%$ & 2195 & $4.52 \%$ & 513 & $32.56 \%$ \\
\hline
\end{tabular}

By running several different simulation scenarios, Table 4 shows the average WIP inventory values of each production line and cycle time with variations in $\boldsymbol{s}$. When $\boldsymbol{s}$ is lower than 15 , resulting from a narrow span of WIP control, the absolute error value $c e$ is also reduced and the correction factors $\alpha_{2}$ does not work properly. This causes a higher WIP level that cannot be reduced quickly, and the results are not satisfactory and largely depart from $s=30$. When $s$ is larger than 45 , conversely, resulting from a wider span of WIP control, the relative error value $\boldsymbol{e}$ is small and the correction factor $\boldsymbol{\alpha}_{1}$ does not work properly. This results in WIP levels close to $s$ not being reduced while higher WIP levels can be reduced quickly; these results are thus slightly higher than $\boldsymbol{s}=30$. The results for $\boldsymbol{s}=40$ are generally better than those for $\boldsymbol{s}=30$. 


\section{Zhao and Takakuwa}

For changes in $\boldsymbol{s}$ ranging from 25 to 45 , the results maintain their stability. This work shows that the optimized model (TO-BE model) has higher robustness and stability levels with a heightened randomicity tolerance capability for stochastic factors. Similarly, other major factors affecting the system reduce sensitivity to control effects. The optimized fuzzy control method thus performs strongly.

\section{CONCLUSIONS}

This paper, aiming to resolve problems in a multi-variety and small-batch production system, has developed a distributed fuzzy controller. It is used to maintain the WIP inventory and cycle times at a low level by checking the inventory levels of distributed WIP buffers and dynamically adjusting the processing rate of each workstation. According to the surplus-based system, using correction factors makes the dynamic real-time WIP inventory level changes close to the hedging point and maintains system stability. The advantage of this two-dimensional fuzzy controller is that it can provide a supervisor group with a control policy based on simple representations and linguistic IF-THEN rules. A VBA module operates all fuzzy calculations for each distributed workstation in the simulation model. By analyzing a system bottleneck tightly coupled cell, a proposed optimized method, which integrates a "Pull"/"Push" mode and fuzzy method, is embedded into the discrete simulation model by fixing specific major stochastic factors. An AS-IS model joined with a TO-BE model provides remarkable control ability for WIP and enhanced cycle time. Noticeable performance improvements and robustness are achieved with this model. This fuzzy control policy thus represents a successful approach to reduce WIP and shorten cycle time for this modern production system.

Future studies will consider a discrete production system with multiple tightly coupled cells, in which the WIP buffer is a shared setting for multi-workstations and the capacity is changeable within certain limits. More stochastic factors will be changed and system flexibility with meeting multi-production lines will also be studied.

\section{ACKNOWLEDGEMENTS}

The authors wish to express their sincere gratitude to Mr. Y. Watanabe of Metal Worker Toa \& Arai Company, Ltd. and Mr. Y. Murata of Fujita Health University, for their cooperation in completing this research. This research was supported by the Grant-in-Aid for the Asian CORE Program "Manufacturing and Environmental Management in East Asia" of the Japan Society for the Promotion of Science (JSPS).

\section{REFERENCES}

Bai, S. X., and S. B. Gershwin. 1994a. "Scheduling Manufacturing Systems with Work-In-Process Inventory Control: Multiple-Part-Type System". International Journal of Production Research Vol.32, No.2:365-386.

Bai, S. X., and S. B. Gershwin. 1994b. "Scheduling Manufacturing Systems with Work-In-Process Inventory Control: Single-Part-Type System”. IIE Transaction Vol.27:599-617.

Diamantidis, A. C., and C. T. Papadopoulos. 2006. "Markovian Analysis of A Discrete Material Manufacturing System with Merge Operations Dependent and Idleness Failures". Computers \& Industrial Engineering Vol.50, No.4:466-487.

Driankov, D., H. Hellendoorn, and M. Reinfrank. 1994. “An Introduction to Fuzzy Control”. Journal of Process Control Vol.4, No.4:298.

Gershwin, S. B. 2000. "Design and Operation of Manufacturing System: The Control Policy". IIE Transactions Vol.32:891-906.

Ioannidis, S., N. C. Tsourveloudis, and K. Valavanis. 2004. "Fuzzy Supervisory Control of Manufacturing Systems". Robotics and Automation Vol.20:379-389.

Jan, A. P., and S. Anders. 2009. "Restricted Work-In-Process: A Study of Differences Between Kanban and CONWIP". International Journal of Production Economics Vol.118, No.1:199-207. 
Kenneth R. B. 1992. "Tightly-Coupled Production Systems: Models, Analysis, and Insights". Journal of Manufacturing Systems Vol.11, No.6:385-400.

Luca, B. 2011. "Determining the Optimal Dimension of a Work-In-Process Storage Area". International Journal of Production Economics Vol.131, No.2:483-489.

Morteza, R. B., J. Morteza, and B. Mehdi. 2011. "Effects of Imperfect Products on Lot Sizing with WorkIn-Process Inventory". Applied Mathematics and Computation V1.217, No.21:8328-8336.

Tamani, K., R. Boukezzoula, and G. Habchi. 2009. "Intelligent distributed and supervised flow control methodology for production systems". Engineering Applications of Artificial Intelligence Vol.22,No.7:1104-1116.

Tamani, K., R. Boukezzoula, and G. Habchi. 2011. "Multi-objective supervisory flow control based on fuzzy interval arithmetic: Application for scheduling of manufacturing systems". Simulation Modeling Practice and Theory Vol.19, No.5:1371-1383.

Tao,W., S. Leyuan, Q. Benjamin, and N. A. Duffie. 2008. "Integrated Dynamic and Model on Coupled Closed-loop Workstation Capacity Controls in a Multi-Workstation Production System". In Proceedings of the 2008 Winter Simulation Conference, Edited by S. J. Mason, R. R. Hill, L. Mönch, O. Rose, T. Jefferson, and J. W. Fowler, 1946-1951. Piscataway, New Jersey: Institute of Electrical and Electronics Engineers, Inc.

Tsourveloudis, N. C., E. Dretoulakis, and S. Ioannidis. 2000. "Fuzzy Work-In-Process Inventory Control of Unreliable Manufacturing Systems". Information Sciences Vol.127, No.1-2:69-83.

Tsourveloudis, N.C. 2010. "On the Evolutionary-Fuzzy Control of WIP in Manufacturing Systems". Neurocomputing Vol.73:648-654.

Yang, T., H. P. Fu, and K. Y. Yang. 2007. "An Evolutionary-Simulation Approach for the Optimization of Multi-Constant Work-In-Process Strategy-A Case Study". International Journal of Production Economics Vol.107, No.1:104-114.

\section{AUTHOR BIOGRAPHIES}

RUN ZHAO is a Ph.D. Student at the Graduate School of Economics and Business Administration at Nagoya University in Japan. He received his B. Sc. degree in Industrial Engineering from the China University of Mining And Technology in 2006 and his M. Sc. degrees in Mechanical Engineering (Industrial Engineering) from Chongqing University in 2009. His research interests include the optimization of manufacturing and work-in-process inventory systems using SIMAN and Simulation with ARENA and Green Production Mode. His email address is zhaorun1982@hotmail.com.

SOEMON TAKAKUWA is a Professor at the Graduate School of Economics and Business Administration at Nagoya University in Japan. He received his B. Sc. and M. Sc. degrees in Industrial Engineering from the Nagoya Institute of Technology in 1975 and the Tokyo Institute of Technology in 1977, respectively. He obtained a Ph.D. in industrial engineering from Pennsylvania State University. He holds a Doctorate of Economics from Nagoya University. He holds a P.E. in Industrial Engineering. His research interests include the optimization of manufacturing and logistics systems, management information systems and simulation analysis on these systems in the context of hospitals. He prepared the Japanese editions of both Introduction to simulation using SIMAN and Simulation with ARENA. He currently serves as a senior staff member at the Department of Hospital Management Strategy and Planning at Nagoya University Hospital. His email address is takakuwa@soec.nagoya-u.ac.jp. 